BRX TH-405

ULB-TH-97/03

\title{
Duality, Self-Duality, Sources and Charge Quantization in Abelian $N$-Form Theories
}

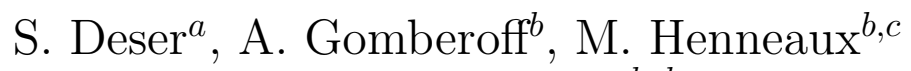 \\ and C. Teitelboim ${ }^{b, d}$ \\ ${ }^{a}$ Department of Physics, Brandeis University, \\ Waltham, MA 02254, U.S.A. \\ ${ }^{b}$ Centro de Estudios Científicos de Santiago, \\ Casilla 16443, Santiago 9, Chile \\ ${ }^{c}$ Faculté des Sciences, Université Libre de Bruxelles, \\ Campus Plaine C.P. 231, B-1050 Bruxelles, Belgium \\ ${ }^{d}$ School of Natural Sciences, Institute for Advanced Study, \\ Princeton, New Jersey 08540, U.S.A.
}

\begin{abstract}
We investigate duality properties of $N$-form fields, provide a symmetric way of coupling them to electric/magnetic sources, and check that these charges obey the appropriate quantization requirements. First, we contrast the $D=4 k$ case, in which duality is a well-defined $\mathrm{SO}(2)$ rotation generated by a Chern-Simons form leaving the action invariant, and $D=4 k+2$ where the corresponding ostensibly $\mathrm{SO}(1,1)$ rotation is not only not an invariance but does not even have a generator. When charged sources are included we show explicitly in the Maxwell case how the usual Dirac quantization arises in a fully symmetric approach attaching strings to both types of charges. Finally, for $D=4 k+2$ systems, we show how charges can be introduced for self-dual $(2 k)$-forms, and obtain the $D=4 k$ models with sources by dimensional reduction, tracing their duality invariance to a partial invariance in the higher dimensions.
\end{abstract}


The ubiquitous current relevance of duality [1] prompts the present investigation, in the simplest context of abelian $N$-form fields. First, we establish a fundamental difference between even and odd $N$-form systems; electricmagnetic duality is only definable for $(2 k+1)$-form potentials, whose (freefield) actions are invariant under this $\mathrm{SO}(2)$ rotation, while the ostensibly hyperbolic $\mathrm{SO}(1,1)$ transformation for $2 k$-forms is not even implementable as a canonical transformation, let alone an invariance. This difference is traceable to the (non-) existence of Chern-Simons terms as generators of duality transformations. We next couple electric/magnetic monopole sources in the specific context of Maxwell theory in Hamiltonian formulation, using a Dirac string approach for both types of charges. We deduce within this context the usual Dirac quantization condition in its manifestly duality invariant form, despite the seeming appearance of the infamous but (spurious) factor 2. Finally, we return to $D=4 k+2$ in the context of self-duality (which in turn is unavailable in $D=4 k$ ). Here we complete previous formulations by introducing coupling to sources. We also show how dimensional reduction to $D=4 k$ yields the manifestly duality-invariant action with sources there and how a partial invariance in the higher space underlies duality invariance in $D=4 k$.

The field strengths are $2 k$ or $(2 k+1)$-forms which we uniformly denote by $F$. For simplicity, we work in flat space but everything carries over to arbitrary backgrounds since there are no covariant derivatives. The basic identities governing the dual operation ${ }^{*} F=\epsilon F$ are

$$
{ }^{* *} F=+F, \quad D=4 k+2 ; \quad{ }^{* *} F=-F, \quad D=4 k .
$$

In the absence of sources, the equations of motion are

$$
d F=0, \quad d^{*} F=0,
$$

and are invariant under linear transformations of $F$ and ${ }^{*} F$,

$$
\left(\begin{array}{c}
F^{\prime} \\
{ }^{*} F^{\prime}
\end{array}\right)=B\left(\begin{array}{c}
F \\
{ }^{*} F
\end{array}\right) \equiv\left(\begin{array}{ll}
a & b \\
c & d
\end{array}\right)\left(\begin{array}{c}
F \\
{ }^{*} F
\end{array}\right), \operatorname{det} B \neq 0 .
$$

In view of (1), the condition that ${ }^{*} F$ is the dual of $F$ imposes both $a=d$ and $c=b(D=4 k+2)$ or $c=-b(D=4 k)$. In the first case, the group defined 
by (3) is $R^{+} \times Z_{2} \times S O(1,1)$ since any invertible $2 \times 2$ matrix with $a=d$ and $c=b$ can be uniquely decomposed as the product of (commuting) factors

$$
B \equiv\left(\begin{array}{ll}
a & b \\
b & a
\end{array}\right)=\left(\begin{array}{cc}
\lambda & 0 \\
0 & \lambda
\end{array}\right)\left(\begin{array}{ll}
0 & 1 \\
1 & 0
\end{array}\right)^{\epsilon}\left(\begin{array}{ll} 
\pm \cosh \alpha & \pm \sinh \alpha \\
\pm \sinh \alpha & \pm \cosh \alpha
\end{array}\right)
$$

for some appropriate $\alpha$. Here, $\lambda=(|\operatorname{det} B|)^{\frac{1}{2}}$ and $\epsilon=0(\operatorname{det} B>0)$ or $\epsilon=1$ (det $B<0$ ). In the second case, the group defined by (3) and $a=d, c=-b$ is $R^{+} \times S O(2)$ since one has

$$
B \equiv\left(\begin{array}{ll}
a & b \\
-b & a
\end{array}\right)=\left(\begin{array}{ll}
\lambda & 0 \\
0 & \lambda
\end{array}\right)\left(\begin{array}{ll}
\cos \alpha & \sin \alpha \\
-\sin \alpha & \cos \alpha
\end{array}\right)
$$

for $\lambda=(\operatorname{det} B)^{\frac{1}{2}}$ and some appropriate $\alpha$.

The above groups are symmetry groups of the equations of motion, with $F$ regarded as the independent variable. However, in order to be true symmetries of the theory, the duality transformations should leave the action invariant. Since the action principle involves the potential $A$ as basic field, $F=d A$ being a derived quantity, this means that one must rewrite the duality transformations in terms of the potential, and verify whether they leave the free field action

$$
I=\int(d x) \operatorname{tr}\left[F F-{ }^{*} F^{*} F\right]
$$

invariant. [We have dropped an irrelevant multiplicative numerical factor and have used (1) as well as $\operatorname{tr} A^{*} B=\operatorname{tr}{ }^{*} A B$, where the trace is on the spacetime indices to write $\operatorname{tr}\left(F F+{ }^{*} F^{*} F\right)=0$ in all dimensions.]

Once the potential is introduced, the equation $d F=0$ becomes an identity, while $d^{*} F=0$ remains an equation of motion. Thus, one cannot rotate $F$ into ${ }^{*} F$ off-shell, and the best that can be achieved is to define the duality transformation of the potential in such a way that the induced transformation of the field strengths reduces to (3) on-shell, with $B$ given by (4) or (5). This task is carried out explicitly below, but the results can be anticipated more quickly by considering the energy-momentum tensor. Because the duality transformations must commute with the Lorentz transformations if they are to be symmetries of the action, they should leave the energymomentum tensor (whose moments generate the Poincaré group) on-shell invariant. [This condition is of course also required for invariance of the gravitational coupling.] 
The stress tensor may be written uniformly in all dimensions as

$$
2 T^{\mu \nu}=F^{\mu} F^{\nu}+{ }^{*} F^{\mu *} F^{\nu}
$$

where the unwritten indices are summed over. Conformal invariance of these theories is reflected in the identical tracelessness of $T^{\mu \nu}$ in all dimensions, remembering that $\operatorname{tr}\left(F^{2}+{ }^{*} F^{2}\right) \equiv 0$. Since the $F F$-term and the ${ }^{*} F^{*} F$-term have the same sign, as required by positive-definiteness of $T_{00}$, it is clear that the energy-momentum tensor is only invariant under $O(2)$ rotations of $F$ into ${ }^{*} F$ and is not invariant under hyperbolic rotations. The subgroups of the above groups that leave $T_{\mu \nu}$ invariant are thus the intersections of (4) or (5) with $O(2)$ and are equal to the factors

$$
G=Z_{2}, \text { for } D=4 k+2
$$

and

$$
G=S O(2), \text { for } D=4 k \text {. }
$$

We shall call these groups the "duality groups". There is therefore an essential difference between the cases $D=4 k+2$ and $D=4 k$. While the duality group is a one-parameter continuous group in the latter, it is a discrete group with just two elements in the former.]

We now verify that these conclusions are correct and that $S O(2)$-duality rotations do indeed leave the action invariant for $D=4 k$, while hyperbolic rotations are not symmetries of the action for $D=4 k+2$. It would be meaningless to transform the $F$ 's formally in the action, since they are not the dynamical variables. As stressed above, in order to discuss any putative symmetry transformation, one must first be able to implement it on the potential $A$. To this end, the Hamiltonian formulation is most convenient; while not manifestly Lorentz invariant, it does preserve manifest gauge invariance. The required discussion was first given long ago [2, 3] for Maxwell theory in four dimensions, which is illustrative of the general $D=4 k$ case.

In the absence of sources, the canonical variables are the transverse, gauge invariant pair $\left(\mathbf{E}_{T}, \mathbf{A}_{T}\right)$. It is convenient to write $\mathbf{E}_{T}=\boldsymbol{\nabla} \times \mathbf{Z}_{T}$ (to parallel

\footnotetext{
${ }^{1}$ Note that in the $D=4 k+2$ case, the $S O(1,1)$ transformation given by minus the unit matrix leaves also the stress tensor invariant. So, the duality group is actually $Z_{2} \times Z_{2}$. However, this additional $Z_{2}$ is rather trivial since it amounts to changing the sign of $A, F$ and ${ }^{*} F$. For this reason, it has not been mentioned in the text.
} 
$\left.\mathbf{B}=\boldsymbol{\nabla} \times \mathbf{A}_{T}\right)$ and then define the 2-vector $\mathbf{A}_{a}=(\mathbf{A}, \mathbf{Z})$ as well as the derived quantities

$$
\mathbf{B}^{a}=\nabla \times \mathbf{A}^{a}, \quad \mathbf{E}^{a}=\dot{\mathbf{A}}^{a},
$$

dropping the " $T$ " notation hereafter. In terms of these, the manifestly $S O(2)$ invariant canonical action becomes]

$$
I=\frac{1}{2} \int d^{4} x\left(\epsilon_{a b} \mathbf{B}^{a} \cdot \mathbf{E}^{b}-\mathbf{B}^{a} \cdot \mathbf{B}^{b} \delta_{a b}\right) .
$$

Note the absence of the Lagrange multipliers $A_{0}^{a}$ (as well of course as of the longitudinal gauge components) since the corresponding Gauss constraints $\boldsymbol{\nabla} \cdot \mathbf{E}=\boldsymbol{\nabla} \cdot \mathbf{B}=0$ are already incorporated. The invariance of (11) under $S O(2)$ rotations

$$
\left(\begin{array}{l}
\mathbf{A}_{1}^{\prime} \\
\mathbf{A}_{2}^{\prime}
\end{array}\right)=\left(\begin{array}{cc}
\cos \alpha & \sin \alpha \\
-\sin \alpha & \cos \alpha
\end{array}\right)\left(\begin{array}{c}
\mathbf{A}_{1} \\
\mathbf{A}_{2}
\end{array}\right)
$$

is obvious since both $\epsilon_{a b}$ and $\delta_{a b}$ are invariant tensors for $S O(2)$. The transformation (12) rotates the $B$ 's (and the $E$ 's) among themselves and reduces to (3) on-shell since $\epsilon_{a b} \mathbf{B}^{b}$ and $\mathbf{E}^{a}$ coincide there.

The invariance of the kinetic term implies that the transformation (12) is a canonical one. The generator that performs the rotations is simply the Chern-Simons term

$$
G=-\frac{1}{2} \int d^{3} x \mathbf{A}^{a} \cdot \mathbf{B}^{b} \delta_{a b}
$$

The invariance of the Hamiltonian is equivalent to $[H, G]=0$. The duality invariance of the stress tensor is likewise obvious, as it depends on either $\mathbf{B}^{a} \mathbf{B}^{a}$ or $\epsilon_{a b} \mathbf{B}^{a} \mathbf{B}^{b}$.

By contrast, the zero-form $(\bmod 2 k)$ potential has canonical action

$$
I=\int d^{2} x\left[E \dot{A}-\frac{E^{2}+A^{\prime 2}}{2}\right]
$$

which seems formally very like its Maxwell analog above. The $S O(1,1)$ transformations read, in terms of the dynamical variables $A$ and $E$ (or equivalently,

\footnotetext{
${ }^{2}$ The action (11) was written in [2] starting directly from the Maxwell action in Hamiltonian form and solving the Gauss law. It was rederived independently in [4]. A manifestly covariant formulation was given recently in [5], but it requires an infinite number of fields.
} 
$A$ and $Z$ with $Z^{\prime}=E$ )

$$
\left(\begin{array}{c}
A_{N E W} \\
Z_{N E W}
\end{array}\right)=\left(\begin{array}{cc}
\cosh \alpha & \sinh \alpha \\
\sinh \alpha & \cosh \alpha
\end{array}\right)\left(\begin{array}{c}
A \\
Z
\end{array}\right)
$$

and are quite similar to (12). However, they do not leave the symplectic (kinetic) term invariant and hence do not define canonical transformations. Indeed, the infinitesimal transformation $\delta A=Z, \delta Z=A$ yields $\delta(E \dot{A})=$ $A^{\prime} \dot{A}+Z^{\prime} \dot{Z}$, which is not a total derivative. The same conclusion would be reached for $S O(2)$ rotations of $A$ and $Z$, since changing the sign of the $Z^{\prime} \dot{Z}$ term in $\delta(E \dot{A})$ does not help. The absence of a canonical generator analogous to (13) for the duality rotations in $4 k+2$ dimensions is of course also clear from the fact that there are no Chern-Simons terms in $1(\bmod 4 k)$ space dimensions for the $2 k$-form spatial component potentials. Note furthermore that $S O(1,1)$ does not even leave the Hamiltonian invariant. We conclude that duality rotations - whether $\mathrm{SO}(1,1)$ or even $\mathrm{SO}(2)$ - are not symmetries of the theory and cannot be implemented as canonical transformations. The $Z_{2}$ transformation of (4) is however a canonical transformation that leaves the Hamiltonian invariant since it exchanges $E$ with $A^{\prime}$; the generating function for that transformation is just $\int d x A_{O L D}^{\prime} A_{N E W}$. One may actually decompose the fields according to the two irreducible representations of $Z_{2}$ (chiral and anti-chiral ( $2 k)$-forms) and write actions for the individual chiral and antichiral components [6, [7]. []

We now turn to the introduction of electric and magnetic sources of the Maxwell field, using Dirac strings [8] symmetrically to describe both, rather than using Coulomb fields for one type and strings for the other. Call particle $A$ 's charge and mass $\left(q_{A}, m_{A}\right)$ and attach a string $y_{A}\left(\sigma_{A}, \tau_{A}\right)$ to it, the range of the spacelike $\sigma_{A}$ being $(0, \infty)$, and $\tau_{A}$ the proper time; a particle's trajectory is specified by $z_{A}^{\mu}\left(\tau_{A}\right)=y^{\mu}\left(\sigma_{A}=0, \tau_{A}\right)$. Instead of Coulomb fields, we add

\footnotetext{
${ }^{3}$ One reaches similar conclusions in a spacetime with Euclidean signature. The signs in (11) are reversed, and the relative sign in the energy-momentum tensor is also changed. The duality group in $4 k$ dimensions is now $S O(1,1)$ and leaves the action invariant. By contrast, duality (whether $S O(2)$ or $S O(1,1)$ ) does not leave the action invariant in $4 k+2$ dimensions. Only $Z_{2}$ is a symmetry. One may construct actions for chiral and anti-chiral $2 k$-forms in $4 k+2$ dimensions, but the chirality condition involves a factor of $i$. The corresponding actions are just the Euclidean continuations of the (first-order) actions of [6, 7].
} 
to the free fields $\left(\mathbf{E}^{a}, \mathbf{B}^{a}\right)$ the following string worldsheet terms:

$$
\begin{aligned}
\mathbf{B}^{a} & =\boldsymbol{\nabla} \times \mathbf{A}^{a}+\sum_{A} \int d y_{A}^{0} \wedge d \mathbf{y}_{A} q_{A}^{a} \delta^{4}\left(x-y_{A}\right), \\
\mathbf{E}^{a} & =\dot{\mathbf{A}}^{a}+\frac{1}{2} \sum_{A} \int d \mathbf{y}_{A} \wedge \times d \mathbf{y}_{A} q_{A}^{a} \delta^{4}\left(x-y_{A}\right)
\end{aligned}
$$

where in (17) there is also a vector cross product indicated. Note that the symmetric Gauss law

$$
\boldsymbol{\nabla} \cdot \mathbf{B}^{a}=\sum q_{A}^{a} \delta^{(3)}\left(x-z_{A}\right)
$$

is implied by (16). The total action is then the sum of the Maxwell action (11) with the redefined fields of (16)-(17) together with the current interaction and free particle contributions,

$$
\begin{aligned}
I_{i s t}= & I_{\text {max }}+I_{\text {int. }}+I_{p}= \\
& \frac{1}{2} \int d^{4} x\left(\mathbf{B}^{a} \cdot \mathbf{E}^{b} \epsilon_{a b}-\mathbf{B}^{a} \cdot \mathbf{B}^{a}\right)+\frac{1}{2} \sum_{A} \epsilon_{a b} q_{A}^{b} \int \mathbf{A}^{a}\left(z_{A}\right) \cdot d \mathbf{z}_{A} \\
- & \sum_{A} m_{A} \int \sqrt{-\left(d z^{\mu}\right)^{2}} .
\end{aligned}
$$

Varying $I_{\text {tot }}$ with respect to $\mathbf{A}^{a}$ gives

$$
\mathbf{B}^{a}+\epsilon_{a b} \boldsymbol{\nabla} \times \mathbf{B}^{b}+\sum_{A} q_{A}^{a} \int \delta^{4}\left(x-z_{A}\right) d \mathbf{z}=0
$$

or equivalently, $\boldsymbol{\nabla} \times\left(\epsilon_{a b} \mathbf{B}^{b}+\mathbf{E}_{a}\right)=0$, which together with (18) are the Maxwell equations with magnetic/electric fields/charges described by $\left(\mathbf{B}^{1}, q_{A}^{1}\right)$, $\left(\mathbf{B}^{2}, q_{A}^{2}\right)$. The particle equations from varying $z_{A}^{\mu}$ yield the correct Lorentz force law,

$$
\begin{aligned}
m \ddot{\mathbf{z}}_{A} & =q_{A}^{a} \mathbf{B}^{a}\left(z_{A}\right) \dot{z}_{A}^{0}+\epsilon_{a b} q_{A}^{a} \mathbf{B}^{b}\left(z_{A}\right) \times \dot{\mathbf{z}}_{A} \\
m \ddot{z}_{A}^{0} & =q_{A}^{a} \mathbf{B}^{a} \cdot \dot{\mathbf{z}}
\end{aligned}
$$

where dots denote derivatives with respect to proper time. There remain the string coordinates, to be varied (leaving the history of the corresponding pole fixed). Properly, this does not lead to any new equations (on Maxwell shell) but consistency requires that no pole of charge $q_{A}^{a}$ cross a string attached to 
any other particle $B$. Note that, in the absence of dyons, i.e., when there are only pure electric and pure magnetic poles - say one of each - our action (19) reduces to the original Dirac form [8] up to a total divergence in field space which enables one to drop the electric string; they are therefore equivalent. Finally, note the unusual $\frac{1}{2}$ factor in the "A · j" term of (19); as we have seen, the correct Lorentz force law nevertheless comes from it together with the string-related contributions implicit in (16)-(17), which bring in another factor of $\frac{1}{2}$.

We are now in a position to derive the usual charge quantization condition in its manifestly duality-invariant form [8, 9, 10]

$$
\epsilon_{a b} \bar{q}^{a} q^{b}=n h
$$

Its validity is most directly noted from the Lorentz force law (21) which involves the $\mathrm{U}(1)$ connections $\mathbf{A}^{a}$, not $\frac{1}{2} \mathbf{A}^{a}$, then following the usual $\mathrm{Wu}^{-}$ Yang arguments [11]. However, it behooves us to show that even at the level of the action (19) the $\frac{1}{2}$ does not mean that a doubling of the right side of (22) is required. The apparent paradox here is similar to the complications as to when a wave function's phase must change by $2 \pi n$ in discussing anyons in $(2+1)$ dimensions. Indeed, the wave functional $\Psi$ 's dependence on the string coordinates $y_{A}$ implies that it acquires a phase $\frac{1}{2 \hbar} \epsilon_{a b} \bar{q}^{a} q^{b}$ as the string of the charge $\bar{q}^{a}$, say, is passed around that of some other $q^{b}$. However, there is no requirement that $\Psi$ be single-valued under this motion because the configuration space is not simply connected; a wave function is perfectly permitted to acquire a phase when the loop in question is not (as is the case in general here) contractible to a point. However, if one does a "double pass" in which the string attached to $\bar{q}^{a}$ is passed around another $q^{b}$, while that of $q^{b}$ is passed around $\bar{q}^{a}$, then that process is connected to the identity, so that the total phase acquired must be a multiple of $2 \pi$. But this double pass is precisely twice the above phase, $2 \times\left(\frac{1}{2 \hbar} \epsilon \bar{q} q\right)$. In the original Dirac formulation there is neither the $\frac{1}{2}$ factor to start with in the interaction, nor a multiply connected configuration space to compensate for it, hence (22) always emerges in both representations, as it must.

Finally, we discuss some new results concerning self-dual systems in $D=$ $4 k+2$ of which the 2 -form potential $A_{\Lambda \Delta}$ in $D=6$ is the first interesting case beyond the $D=2$ chiral scalar field. Here we extend the original non- 
manifestly Lorentz covariant formulation [7] by introducing sources.f We will also reduce the 2 -form action to Maxwell by dimensional reduction and obtain directly the manifestly duality invariant action (19). In the process, we will trace the sources of Maxwell duality invariance to a particular rotation in the higher dimensional space.

The field strengths are defined as usual as

$$
F_{\Gamma \Delta \Lambda}=\partial_{\Gamma} A_{\Delta \Lambda}+\partial_{\Delta} A_{\Lambda \Gamma}+\partial_{\Lambda} A_{\Gamma \Delta} .
$$

The sourcefree action for a chiral 2-form is given by the first-order expression [7]

$$
I=\frac{1}{4} \int d^{6} x\left[E^{A B} B_{A B}-B^{A B} B_{A B}\right]
$$

where the electric and magnetic components $E^{A B}$ and $B^{A B}$ are defined through

$$
\begin{aligned}
E^{A B} & =-F^{0 A B}, \\
B^{A B} & =\frac{1}{6} \epsilon^{A B C D E} F_{C D E} .
\end{aligned}
$$

Here, capital roman letters take the spatial values $(a, 4,5)$ with $a=1,2,3$. Similarly, we introduce capital greek indices with values $\Lambda=(\lambda, 4,5), \lambda=$ $0,1,2,3$. The separation-out of two spatial directions $(4,5)$ is motivated by the reduction to four dimensions performed below. The equations of motion are obtained by varying the action with respect to the 2-form components $A_{A B}\left(A_{0 A}\right.$ drops out) and are equivalent to the self-duality condition

$$
E^{A B}=B^{A B} \text {. }
$$

It is possible to introduce sources while maintaining the chirality condition (27), provided these sources have equal magnetic and electrical charges. That is, the electric and magnetic currents must be equal,

$$
J_{e}^{\Lambda \Delta}=J_{m}^{\Lambda \Delta} \equiv J^{\Lambda \Delta} .
$$

\footnotetext{
${ }^{4}$ The difficulty of writing a finite-component self-dual covariant form is simply that because of the identity $F^{2}=-{ }^{*} F^{2}$, the (anti) self duality condition ${ }^{*} F= \pm F$ when squared implies ${ }^{*} F^{2}=+F^{2}$ and thus $F^{2}=0$. Since $F^{*} F$ is a total derivative, there is no non trivial covariant expression that is quadratic in the field strength. Some attempts to bypass this difficulty by introducing auxiliary fields - in most cases an infinite number of them - are described in [5, 12, 13, 14].
} 
The action describing the coupling is obtained from the free action (24) by (i) adding to it the minimal coupling term $A_{\Lambda \Delta} J^{\Lambda \Delta}$; and (ii) redefining the fields $E^{A B}$ and $B^{A B}$ by including a contribution from the sources and such that $B^{A B}$ fulfills

$$
\partial_{B} B^{A B} \equiv J^{0 A}
$$

(and no longer $\partial_{B} B^{A B} \equiv 0$ ) identically. Explicitly,

$$
\begin{aligned}
E^{A B} & =-F^{0 A B}+\frac{1}{6} \epsilon^{A B C D E} G_{C D E} \\
B^{A B} & =\frac{1}{6} \epsilon^{A B C D E} F_{C D E}-G^{0 A B}
\end{aligned}
$$

where $F_{\Gamma \Delta \Lambda}$ is still given by (23) and where $G_{\Gamma \Delta \Lambda}$ has Dirac-string type singularities and is completely determined by the sources through

$$
\partial_{\Lambda} G^{\Gamma \Delta \Lambda}+J^{\Gamma \Delta}=0
$$

(see below). So, the action is

$$
I=\frac{1}{4} \int d^{6} x\left[E^{A B} B_{A B}-B^{A B} B_{A B}-A_{\Lambda \Omega} J^{\Lambda \Omega}\right] .
$$

The temporal components $A_{0 B}$ again drop out and the equations of motion obtained by varying the spatial components $A_{A B}$ are equivalent to the chirality condition (27).

If one takes as source the elementary object to which the 2-form naturally couples, namely, a charged string, then $J^{\Lambda \Omega}$ is given by

$$
J^{\Lambda \Omega}(x)=e \int_{W S} \delta^{(6)}(x-z) d z^{\Lambda} \wedge d z^{\Omega}
$$

where the integral is taken along the string world sheet $z^{\Lambda}(\tau, \sigma)$ and where $e=g$ is the electric (= magnetic) strength of the string. The field $G_{\Gamma \Delta \Lambda}$ has support on a membrane emanating from the string and is given by [15]

$$
G^{\Gamma \Delta \Lambda}(x)=e \int_{M W S} \delta^{(6)}(x-y) d y^{\Gamma} \wedge d y^{\Delta} \wedge d y^{\Lambda}
$$

Here, the integral is taken over the world sheet of the membrane $y^{\Lambda}(\tau, \sigma, \rho)$ with $0 \leq \rho<\infty$ and $y^{\Lambda}(\tau, \sigma, 0)=z^{\Lambda}(\tau, \sigma)$. Varying the action, including the 
string's kinetic term, with respect to the string coordinates yields the string equations of motion, with the appropriate Lorentz force. Varying the action with respect to the membrane coordinates yield no equation, provided the membrane does not intersect any other string ("Dirac veto"). In the quantum regime, one finds that the string electric (= magnetic) strength $e$ fulfills the quantization condition

$$
e g \equiv e^{2}=n h \text {. }
$$

This condition can be derived either à la Wu-Yang [11, 16], or by requiring as above that the membrane remain quantum-mechanically unobservable [15, 17]. It has been used recently in [18]. One striking feature of (36) is that it is not invariant under $S O(2)$ or $S O(1,1)$ rotations of the electric and magnetic charges (the product $e g$ is not invariant). But this is all right since neither $S O(1,1)$ nor $S O(2)$ duality is a symmetry of the action in six dimensions. Only $Z_{2}$ is, and the quantization condition remains clearly unchanged if one interchanges (the equal-valued) $e$ and $g$.

If the spacetime has the topology $R^{4} \times T^{2}$, with two spatial coordinates compactified on a torus $T^{2}$, one may naturally consider sources of higher dimensions, here membranes that are wrapped on the torus. These extended objects thus have one more dimension than the elementary source to which the 2-form couples and correspond, say, to charged rings in electromagnetism. We take the coordinates $x^{4}$ and $x^{5}$ to be along the torus. The spacetime history of the membrane is $x^{\mu}=z^{\mu}(\tau), x^{4}=x^{4}, x^{5}=x^{5}$ (we take $\tau, x^{4}$ and $x^{5}$ as parameters). The current does not depend on the internal coordinates $x^{4}, x^{5}$ and is assumed to be of the form

$$
\begin{aligned}
J^{\lambda \mu} & =0, \\
J^{45} & =0, \\
J^{4 \lambda} & =g^{4} \int_{W} \delta^{4}\left(x^{\mu}-z^{\mu}\right) d z^{\lambda}, \\
J^{5 \lambda} & =g^{5} \int_{W} \delta^{4}\left(x^{\mu}-z^{\mu}\right) d z^{\lambda},
\end{aligned}
$$

where $\mathrm{W}$ is the trajectory $x^{\mu}=z^{\mu}(\tau)$ traced out by the membrane in physical space. One may think of the membrane as formed out of strings circling around the fourth and fifth directions. The parameters $g^{4}$ and $g^{5}$ in (40) characterize the density of strings in each direction. The above currents (being those of a particle) are automatically conserved for any choice of $g^{4}$ 
and $g^{5}$. The field $G_{\Lambda \Gamma \Omega}$ has support on a four-dimensional surface which may be taken to be a string worldsheet $y^{\lambda}(\tau, \sigma)$ ending on the worldline $z^{\mu}(\tau)$ in $R^{4}$ times the torus (which we take to have unit volume). Its non-vanishing components do not depend on $x^{4}$ and $x^{5}$ and read

$$
\begin{aligned}
G^{4 \lambda \mu} & =-g^{4} \int_{W S} \delta^{4}\left(x^{\mu}-y^{\mu}\right) d y^{\lambda} \wedge d y^{\mu} \\
G^{5 \lambda \mu} & =-g^{5} \int_{W S} \delta^{4}\left(x^{\mu}-y^{\mu}\right) d y^{\lambda} \wedge d y^{\mu} .
\end{aligned}
$$

Now, if we assume that $A_{\Lambda \Omega}$ does not depend on the torus coordinates $x^{4}$ and $x^{5}$, the action (33) becomes a four-dimensional integral since the integrand does not depend on the internal coordinates. Assuming furthermore that the only non-vanishing components of $A_{\Lambda \Omega}$ are $A_{4 i}$ and $A_{5 i}$ and making the identifications

$$
\begin{aligned}
A_{4 i} & =A_{i}^{1}, \quad A_{5 i}=-A_{i}^{2}, \\
g^{4} & =q^{2}, \quad g^{5}=q^{1}
\end{aligned}
$$

one finds that (33) reduces exactly to the manifestly duality-invariant action (19) for electromagnetism, once one has of course added or reduced to $D=4$ the appropriate kinetic term for the source. From this dimensional reduction perspective, duality appears as a spacetime transformation (rotation in the "internal" 4-5 space). That duality in four dimensions can be obtained from the chiral 2-form in six dimensions upon appropriate dimensional reduction was previously observed in the sourceless case [19, 20, 21].

A more detailed report of the present work will be presented elsewhere [22]. There, we will also investigate duality in the more general context of non-linear electrodynamics and show how the covariant equations determining duality-invariant theories [23] arise in the Hamiltonian formalism through the Dirac-Schwinger Lorentz invariance criterion on the commutator of the equal-time Hamiltonian densities.

\section{Acknowledgements}

The work of SD was supported by the National Science Foundation, grant \#PHY-9315811, that of MH was partly supported by a research grant from FNRS (Belgium), that of AG and CT by Grants 3960008 and 1940203 
of FONDECYT (Chile). AG and CT also acknowledge institutional support to the Centro de Estudios Científicos de Santiago provided by SAREC (Sweden) and a group of Chilean private companies (EMPRESAS CMPC, CGE, COPEC, MINERA LA ESCONDIDA, NOVAGAS Transportandores de Chile, ENERSIS, BUSINESS DESIGN ASS., XEROX Chile).

\section{References}

[1] For a recent review, see D. I. Olive, Exact Electromagnetic Duality, hep-th/9508089.

[2] S. Deser and C. Teitelboim, Phys. Rev. D 13 (1976) 1572; S. Deser, M. Henneaux and C. Teitelboim, Phys. Rev. D 55 (1997) 268.

[3] Some of the free field results given above were recently summarized in S. Deser, Black Hole Electromagnetic Duality, hep-th/9701157.

[4] J.H. Schwarz and A. Sen, Nucl. Phys. B 411 (1994) 35.

[5] N. Berkovits, Phys. Lett. B 388 (1996) 743; Local Actions with Electric and Magnetic Sources, hep-th/9610134.

[6] R. Floreanini and R. Jackiw, Phys. Rev. Lett. 59 (1987) 1873.

[7] M. Henneaux and C. Teitelboim, Phys. Lett. 206 B (1988) 650.

[8] P.A.M. Dirac, Proc. Roy. Soc. London A 133 (1931) 60; Phys. Rev. 74 (1948) 817.

[9] J. Schwinger, Phys. Rev. 173 (1968) 1536; Science 165 (1969) 757.

[10] D. Zwanziger, Phys. Rev. 176 (1968) 1489.

[11] T. T. Wu and C. N. Yang, Phys. Rev. D 12 (1975) 3845; Nucl. Phys. B 107 (1976) 365.

[12] F. P. Devecchi and M. Henneaux, Phys. Rev. D 54 (1996) 1606.

[13] I. Bengtsson and A. Kleppe, On Chiral P-Forms, hep-th/9609102. 
[14] P. Pasti, D. Sorokin and M. Tonin, On Lorentz Invariant Actions for Chiral P-Forms, hep-th/9611100.

[15] C. Teitelboim, Phys. Lett B 167 (1986) 63, 67.

[16] M. Henneaux and C. Teitelboim, Found. Phys. 16 (1986) 593.

[17] R. Nepomechie Phys. Rev. D 31 (1985) 1921.

[18] M. Perry and J.H. Schwarz, Interacting Chiral Gauge Fields in Six Dimensions and Born-Infeld Theory, hep-th/9611065.

[19] E. Verlinde, Nucl. Phys. B 455 (1995) 211.

[20] D. Berman, Classical Duality from Compactification of Self-Dual Five Form Maxwell Theory in Ten Dimensions, hep-th/9612191.

[21] I. Giannakis and V. P. Nair, Symplectic Structures and Self-Dual Fields in $(4 k+2)$ Dimensions, hep-th/9702024.

[22] S. Deser, A. Gomberoff, M. Henneaux, Ö. Sarıoğlu and C. Teitelboim, in preparation.

[23] G. W. Gibbons and D. A. Rasheed, Nucl. Phys. B 454 (1995) 185; I. Bengtsson, Manifest Duality in Born-Infeld Theory, hep-th/9612174. 